\title{
Treatment of a Child With Submassive Pulmonary Embolism Associated With Hereditary Spherocytosis Using Ultrasound-Assisted Catheter-Directed Thrombolysis
}

\author{
Marvin Kajy, MD, ${ }^{1}$ Nimrod Blank, MD, ${ }^{2}$ M. Chadi Alraies, MD, ${ }^{2}$ Jyothsna Akam-Venkata, MD, ${ }^{3}$ \\ Sanjeev Aggarwal, MD, ${ }^{3}$ Amir Kaki, MD, ${ }^{4}$ Tamam Mohamad, MD, ${ }^{2}$ Mahir Elder, MD, ${ }^{2}$ Theodore Schreiber, MD ${ }^{4}$ \\ ${ }^{1}$ Department of Internal Medicine, Wayne State University School of Medicine, Detroit Medical Center Heart Hospital, Detroit, MI \\ ${ }^{2}$ Department of Interventional Cardiology, Wayne State University School of Medicine, Detroit Medical Center, Detroit MI ${ }^{3}$ Department of \\ Cardiology, Wayne State University School of Medicine, Children's Hospital of Michigan, Detroit, MI ${ }^{4}$ Department of Interventional \\ Cardiology, Ascension St. John Hospital, Detroit, MI
}

Background: The clinical presentation of hereditary spherocytosis varies from no symptoms to severe hemolytic anemia requiring splenectomy. Splenectomy imposes the risk of hypercoagulability and acute pulmonary embolism. Catheter-directed thrombolysis is an established treatment for submassive pulmonary embolism in adults. However, the literature regarding its use in children is limited.

Case Report: We present the case of a 12-year-old male with hereditary spherocytosis who was diagnosed with pulmonary embolism and successfully treated with catheter-directed thrombolysis. The patient was initially treated with $10.5 \mathrm{mg}$ of recombinant tissue plasminogen activator ( $\mathrm{r}$-tPA) delivered over 8 hours. However, because of minimal clinical and hemodynamic improvement, a second course of thrombolytic was administered for an additional 24 hours ( $25 \mathrm{mg}$ of $\mathrm{r}$-tPA), and the treatment resulted in marked clinical and hemodynamic improvement. Clot resolution was confirmed via angiography. The patient was discharged on enoxaparin and with regular follow-up. One year later, the patient was asymptomatic on enoxaparin.

Conclusion: This case demonstrates that catheter-based treatment of submassive pulmonary embolism restores hemodynamic stability and thus is an alternative to surgery or systemic thrombolysis, even in the pediatric setting. While catheter-directed thrombolysis is a safe and effective alternative to systemic thrombolysis, further research is needed to establish appropriate dosing and indications in the adolescent population.

Keywords: Endovascular procedures, mechanical thrombolysis, pulmonary embolism, spherocytosis-hereditary, thrombolytic therapy, tissue plasminogen activator, vascular access devices

Address correspondence to M. Chadi Alraies, MD, Department of Interventional Cardiology, Wayne State University School of Medicine, Detroit Medical Center Heart Hospital, 311 Mack Ave., Detroit, MI 48201. Tel: (216) 255-0008. Email: alraies@hotmail.com

\section{INTRODUCTION}

Hereditary spherocytosis is an inherited disorder of the erythrocyte membrane, caused by a variety of molecular defects in the genes that code for the red blood cell structural and membrane proteins, such as ankyrin and spectrin. ${ }^{1}$ Clinical presentation can vary from totally asymptomatic to hemolytic anemia. ${ }^{2}$ Severe hemolysis requires splenectomy to relieve symptoms attributable to anemia or splenomegaly, to reverse growth failure or skeletal changes resulting from overrobust erythropoiesis, and to prevent recurrent gallstones. ${ }^{3}$ However, splenectomy confers a state of hypercoagulability, and patients may develop thromboembolic events such as pulmonary emboli. ${ }^{4-6}$
The incidence of pulmonary embolism in children is very low, $<1$ in $100,000 .{ }^{7}$ No specific treatment algorithms have been developed for management of pulmonary embolism in children, but traditional treatment options have been anticoagulation, thrombolysis, and thrombectomy. To aid in management, the Pulmonary Embolism Severity Index (PESI) and the simplified Pulmonary Embolism Severity Index (sPESI) are risk stratification tools based on clinical factors that can estimate the mortality of patients with pulmonary embolism and help guide treatment (Table 1). Patients with low scores can be treated on an outpatient basis, whereas patients with high scores require escalation of care and immediate intervention. ${ }^{8,9}$ For low-risk pediatric patients, 
Table 1. Calculation of the Pulmonary Embolism Severity Index (PESI) and Simplified Pulmonary Embolism Severity Index (sPESI) Scores ${ }^{8,9}$

\begin{tabular}{lcc}
\hline \multicolumn{1}{c}{ Parameter } & PESI Points & sPESI Points \\
\hline Age & Age in years & +1, if age $>80$ years \\
History of cancer & +30 & +1 \\
History of heart failure & +10 & +1 \\
History of chronic lung disease & +10 & +1 \\
Heart rate $\geq 110$ bpm & +20 & +1 \\
Systolic blood pressure $<100 \mathrm{mmHg}$ & +30 & +1 \\
Respiratory rate $\geq 30$ breaths per minute & +20 & - \\
Temperature $<36^{\circ} \mathrm{C} / 96.8^{\circ} \mathrm{F}$ & +20 & - \\
Altered mental status & +60 & - \\
Oxygen saturation $<90 \%$ & +20 & +1 \\
\hline
\end{tabular}

\section{Risk Stratification}

PESI Score Total Points

$\begin{array}{lcr}0-65 & \text { I } & 0.0 \%-1.6 \% \\ 66-85 & \text { II } & 1.7 \%-3.5 \% \\ 86-105 & \text { III } & 3.2 \%-7.1 \% \\ 106-125 & \text { IV } & 4.0 \%-11.4 \% \\ >125 & \text { V } & 10 \%-24.5 \%\end{array}$

Note: Patients with low scores (PESI $\leq 85$ and SPESI 0 ) may be treated as an outpatient basis, whereas patients with high scores (PESI $>86$ and sPESI $>1$ ) require escalation of care and immediate intervention.

anticoagulation may be instituted to prevent clot progression. For patients who are hemodynamically unstable, systemic thrombolysis may be used. In patients who have contraindications to systemic thrombolysis, mechanical or surgical thrombectomy may be implemented. ${ }^{10}$

Pulmonary embolism is classified as massive (high risk), submassive (intermediate risk), and low risk. The US Food and Drug Administration has approved alteplase, also known as recombinant tissue plasminogen activator ( $r$-tPA), for the treatment of acute massive pulmonary embolism. ${ }^{11}$ The most widely suggested systemic thrombolysis regimen for massive pulmonary embolism in adults is $100 \mathrm{mg}$ of alteplase infused over 2 hours. ${ }^{12}$ The optimal dose of alteplase for systemic thrombolysis in children is not well established, and dosing is based on extrapolation from case series and adult treatment protocols. Because of the uncertainty of benefit vs the risk of massive and life-threatening bleeding, systemic thrombolysis for submassive pulmonary embolism is not indicated (class IIB/level of evidence $C$ by most guidelines). ${ }^{13}$

Catheter-directed thrombolysis has emerged as a possibly safer alternative to systemic thrombolysis in the treatment of submassive pulmonary embolism and is suggested for patients with massive pulmonary embolism who have contraindications to systemic thrombolysis, have failed thrombolysis, or have acute right ventricular dysfunction and cardiogenic shock, likely leading to death before systemic thrombolysis can take effect. ${ }^{14,15}$

We present the case of an adolescent male with hereditary spherocytosis status post splenectomy, who sustained a submassive pulmonary embolism.

\section{CASE REPORT}

A 12-year-old male with hereditary spherocytosis who had a splenectomy at age 6 years presented with right-sided chest pain, hemoptysis, and dyspnea for 1 day. Upon admission, he had a blood pressure of $122 / 63 \mathrm{mmHg}$, heart rate of $128 \mathrm{bpm}$, and pulse oximetry saturation of $87 \%$ on room air. The patient weighed $89 \mathrm{~kg}$ and was $1.55 \mathrm{~m}$ tall (body mass index of $36.9 \mathrm{~kg} / \mathrm{m}^{2}$ ). Physical examination was remarkable for obvious distress, chest retractions, orthopnea, and right upper quadrant tenderness. Computed tomography (CT) of the thorax revealed a large thrombus in the right main pulmonary artery extending into the segmental branches of the right upper, middle, and lower lobes and dilatation of the RV (right ventricle). RV strain was evident, with an 


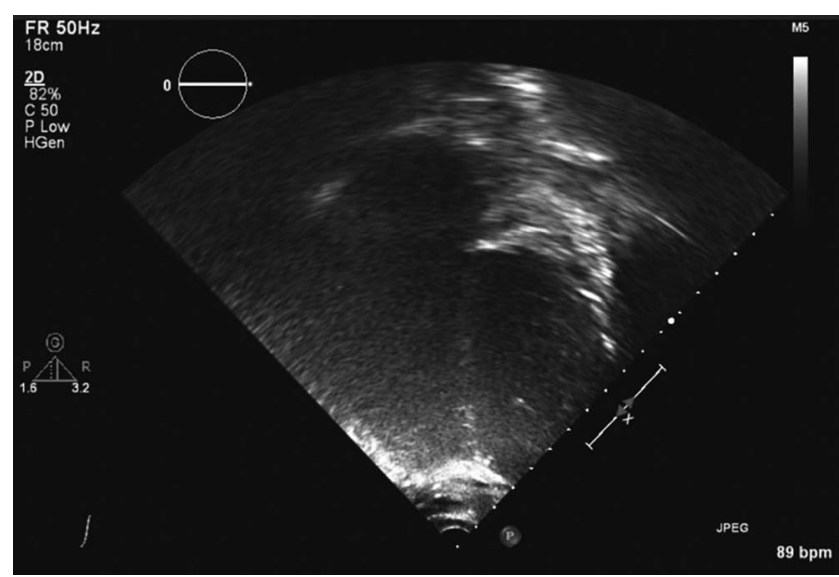

Figure 1. Admission echocardiogram demonstrates massive right ventricular dilatation.

$\mathrm{RV} /$ left ventricle (LV) ratio of 1.15. Troponin I was elevated at $0.069 \mathrm{ng} / \mathrm{mL}$. Echocardiogram revealed moderate right atrial and RV dilation with decreased RV systolic function (Figure 1). LV systolic function appeared normal to hyperdynamic with an ejection fraction of $70 \%-75 \%$. The patient's PESI score was 92, which is class III intermediate risk, and his SPESI score was 8.9 , which is high risk for this patient.

The patient's treatment began with a $20 \mathrm{U} / \mathrm{kg} / \mathrm{hr}$ heparin infusion. Because the patient was considered to have a high-risk submassive $\mathrm{PE}$, he was referred for right heart catheterization and possible catheter-directed thrombolysis. Right heart catheterization revealed severely elevated pulmonary artery pressures $(58 / 25 \mathrm{mmHg}$, mean $39 \mathrm{mmHg}$ ) and elevated RV pressures (Table 2). Pulmonary angiogram revealed a large thrombus in the right pulmonary artery.

Our institutional protocol mandates performing right heart catheterization and pulmonary angiography in patients who have a submassive pulmonary embolism. Upon confirmation of increased RV systolic pressures, treatment with catheter-directed thrombolysis is initiated using the EkoSonic Endovascular System (BTG International Inc.) with 1 or 2 catheters, depending on clot location. In adults, $5 \mathrm{mg}$ of $r$-tPA is administered selectively as a bolus, followed by infusion of r-tPA at rate of $2 \mathrm{mg} / \mathrm{hr}$ for the first 2 hours, followed by $1 \mathrm{mg} / \mathrm{hr}$ for an additional 6 hours.

The EkoSonic Endovascular System is a novel technology that is used to augment catheter-directed thrombolysis. A catheter-covered ultrasound microfilament (12-30 cm long) is selectively placed through the thrombus in the pulmonary artery. Ultrasonic waves generate thrombin microfractures that augment the effects of low-dose thrombolysis, with r-tPA selectively administered through the device catheter. This system is minimally invasive and allows for localized delivery of r-tPA, thereby reducing the risk of bleeding by lowering the total thrombolytic dose delivered. This finding was demonstrated by the ULTIMA (ULTrasound Accelerated Thrombolysls of PulMonAry Embolism) and the SEATTLE II (Submassive and Massive Pulmonary Embolism Treatment With Ultrasound Accelerated Thrombolysis Therapy) trials. ${ }^{16,17}$

In our patient, catheter-directed thrombolysis was started by placing a $12 \mathrm{~cm}$ EkoSonic catheter into the main and middle right pulmonary artery. A $2.5 \mathrm{mg}$ bolus of $\mathrm{r}$-tPA was administered selectively, followed by a continuous $1 \mathrm{mg} / \mathrm{hr}$ r-tPA infusion for 8 hours, yielding a total r-tPA dose of $10.5 \mathrm{mg}$.

After the initial treatment course, echocardiogram showed evidence of only partial improvement. The patient was still tachycardic (heart rate of 110-120 bpm) and hypoxic (saturating $92 \%$ on room air). Echocardiogram showed persistently elevated right heart pressures and mild to no improvement in RV size and function (Figure 2).

A second right heart catheterization showed a persistent large thrombus in the right pulmonary artery with

Table 2. Cardiac Catheterization Measurements at Each Treatment Timepoint

\begin{tabular}{|c|c|c|c|c|c|c|}
\hline Treatment Timepoint & $\begin{array}{l}\text { Right Atrial } \\
\text { Pressure, } \\
\text { mmHg }\end{array}$ & $\begin{array}{l}\text { Right } \\
\text { Ventricular } \\
\text { Pressure, } \\
\text { mmHg }\end{array}$ & $\begin{array}{l}\text { Pulmonary } \\
\text { Artery } \\
\text { Pressure, } \\
\text { mmHg }\end{array}$ & $\begin{array}{l}\text { Blood } \\
\text { Pressure, } \\
\text { mmHg }\end{array}$ & $\begin{array}{c}\text { Heart } \\
\text { Rate, bpm }\end{array}$ & $\begin{array}{c}\text { Pulmonary } \\
\text { Artery } \\
\text { Saturation, \% }\end{array}$ \\
\hline $\begin{array}{l}\text { First cardiac catheterization, } \\
\text { before the first cycle of } \\
\text { EkoSonic Endovascular System } \\
\text { catheter-directed thrombolysis }\end{array}$ & $16 / 17 / 16$ & $59 / 25 / 26$ & $58 / 25 / 39$ & $71 / 58 / 63$ & 107 & $\mathrm{~N} / \mathrm{A}$ \\
\hline $\begin{array}{l}\text { Second cardiac catheterization, } \\
\text { before the second cycle of } \\
\text { EkoSonic Endovascular System } \\
\text { catheter-directed thrombolysis }\end{array}$ & $8 / 7 / 6$ & $53 / 6 / 10$ & $55 / 28 / 39$ & $113 / 53 / 74$ & 100 & 59 \\
\hline $\begin{array}{l}\text { Third cardiac catheterization, } \\
\text { after } 2 \text { cycles of EkoSonic } \\
\text { Endovascular System } \\
\text { catheter-directed thrombolysis }\end{array}$ & $9 / 8 / 5$ & $41 / 0 / 5$ & $41 / 16 / 25$ & $113 / 65 / 81$ & 91 & 72 \\
\hline
\end{tabular}

Note: Right atrial pressure, right ventricular pressure, pulmonary artery pressure, and blood pressure are presented as systolic pressure/diastolic pressure/mean pressure.

$\mathrm{N} / \mathrm{A}$, not applicable. 


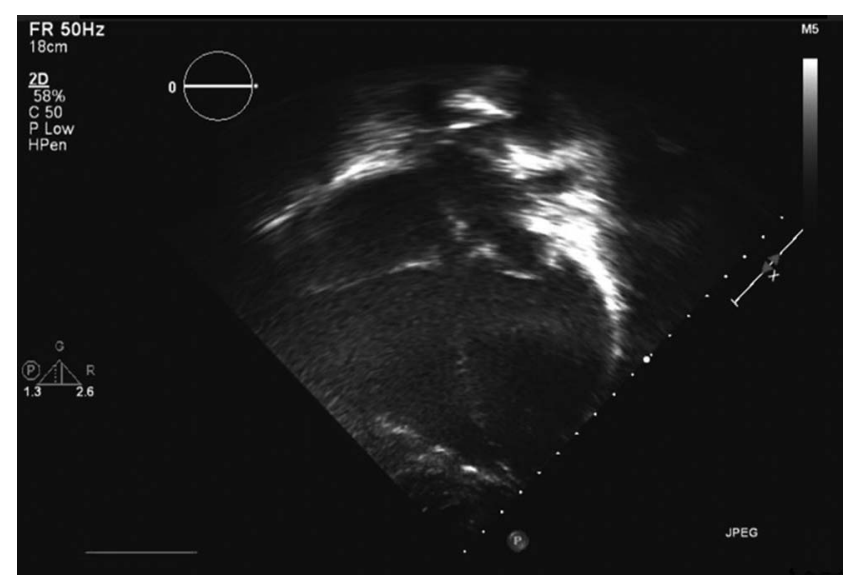

Figure 2. Echocardiogram after completion of the first cycle of EkoSonic Endovascular System catheter-directed thrombolysis demonstrates mild resolution, but the right ventricle is still dilated.

mild to no improvement in pressures, with a pulmonary artery pressure of 55/28 $\mathrm{mmHg}$, mean $39 \mathrm{mmHg}$ (Table 2). Angiogram revealed a large, persistent thrombus in the right pulmonary artery (Figure 3). A second course of catheterdirected thrombolysis was administered using a similar $12 \mathrm{~cm}$ EkoSonic catheter. A total of $25 \mathrm{mg}$ of $\mathrm{r}$-tPA was administered: $2 \mathrm{mg}$ during the first hour, followed by $1 \mathrm{mg} / \mathrm{hr}$ for the next 23 hours.

Follow-up right heart catheterization confirmed marked hemodynamic improvement, with pulmonary artery pressure of $41 / 16 \mathrm{mmHg}$, mean $25 \mathrm{mmHg}$ (Table 2). Pulmonary angiogram showed markedly improved flow to the pulmonary artery.

Clinically, the patient had resolution of hypoxia and tachycardia. He did not experience any bleeding throughout the 2

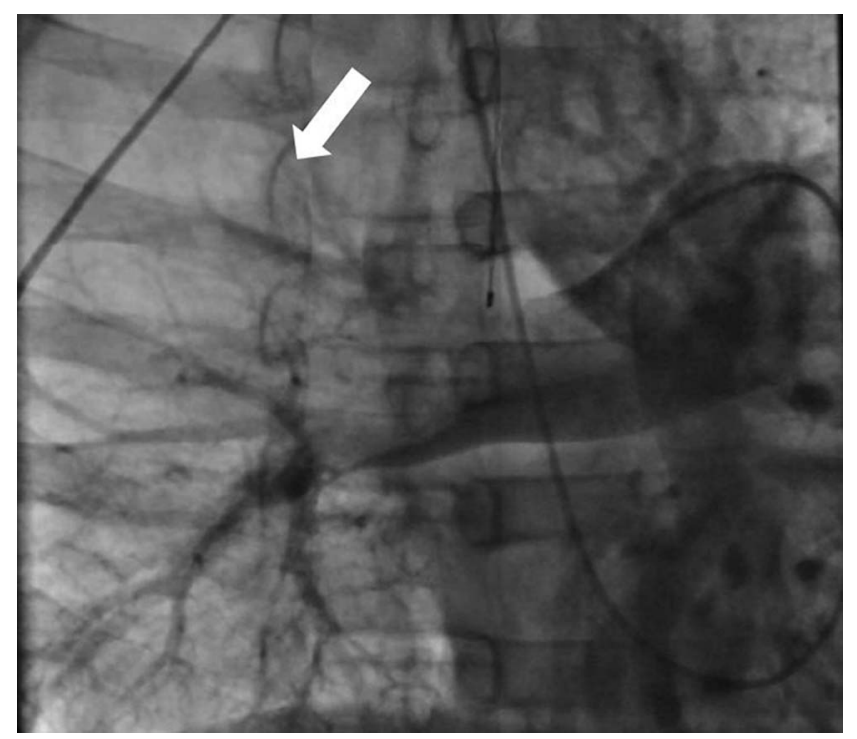

Figure 3. Angiogram after completion of the initial cycle of EkoSonic Endovascular System catheter-directed thrombolysis shows the pulmonary embolism (arrow) with distal oligemia.

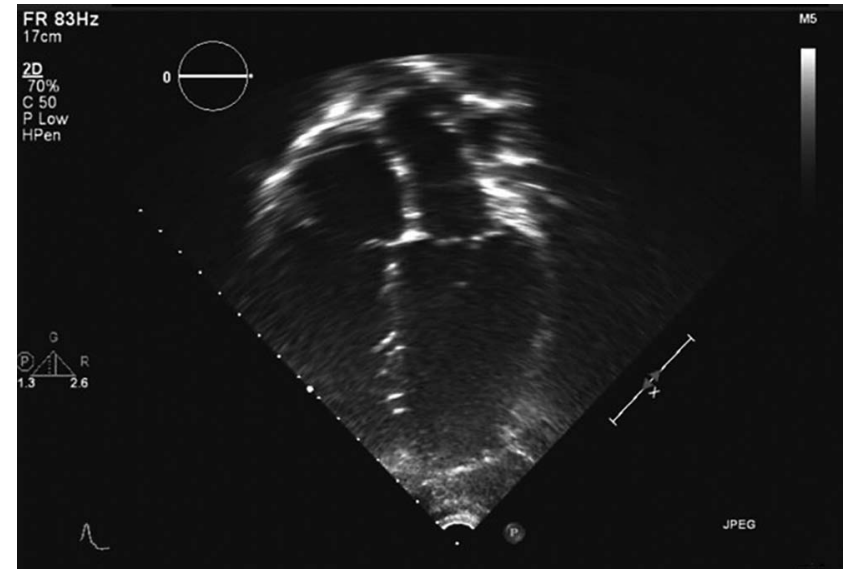

Figure 4. Echocardiogram after completion of the second cycle of EkoSonic Endovascular System catheter-directed thrombolysis demonstrates improvement in right ventricular dilatation.

courses of catheter-directed thrombolysis. Repeat echocardiograms at the completion of catheter-directed thrombolysis therapy and at the time of discharge demonstrated normalization of RV size and resolution of RV systolic function (Figures 4 and 5). Anticoagulation was converted to enoxaparin $80 \mathrm{mg}$ twice daily, and the patient was discharged home after 8 days of hospitalization.

He has had regular follow-up with our hematology clinic for more than 1 year. The patient has not experienced any bleeding events or new thrombotic events while on enoxaparin. Cardiac CT performed after 1 year of anticoagulation revealed near-complete resolution of the pulmonary embolism with no evidence of heart strain. The patient will require lifelong anticoagulation because of his hypercoagulable state. The patient may be transitioned to direct oral anticoagulants (DOACs) in the future. At the time this report was written, the patient was only 13 years old, and no published results about DOAC use in the adolescent population were available at that time.

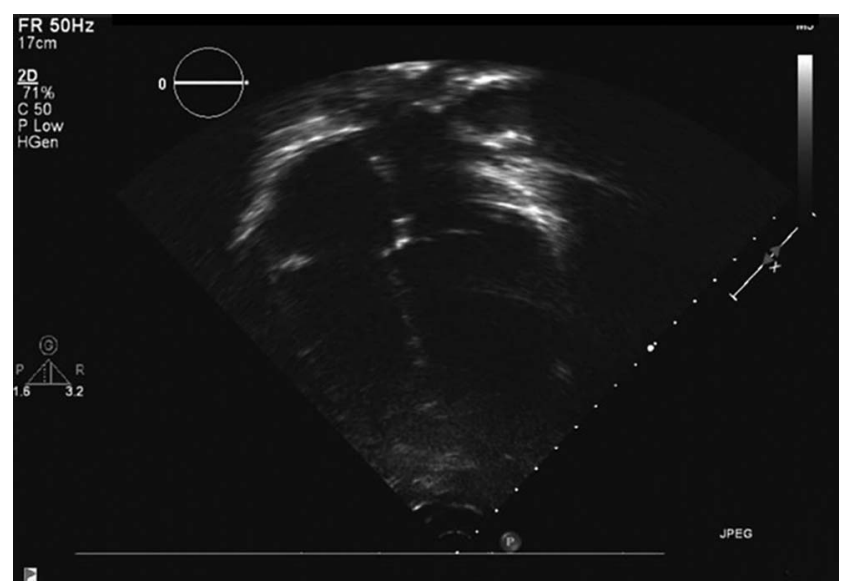

Figure 5. Discharge echocardiogram shows marked improvement of right ventricular size after resolution of the clot. 


\section{DISCUSSION}

Our case has several interesting aspects: the development of a pulmonary embolism following a splenectomy, the use of ultrasound-assisted thrombolysis in the pediatric setting, and the extended duration of ultrasound-assisted thrombolysis.

The existence of a prothrombotic state in patients with hereditary spherocytosis who have been splenectomized has been described in previous publications. ${ }^{4-6,18}$ Hypercoagulability workup was unremarkable for our patient, and he did not have modifiable risk factors for pulmonary embolism. Hypercoagulability in a postsplenectomy state may be attributed to several factors such as polycythemia and thrombocytosis that lead to increased blood viscosity. ${ }^{19}$ In addition, patients without a spleen have an increased number of circulating red blood cells. Damaged red blood cells biochemically enhance platelet activation and aggregation, predisposing to thrombus formation. ${ }^{20}$ Therefore, we speculate that the therapeutic splenectomy for this patient might have been a risk factor for the development of pulmonary embolism. The knowledge that a splenectomized state increases the chance of thromboembolic phenomena raises the interesting question of whether such individuals should be treated with prophylactic anticoagulation.

Anticoagulation is the cornerstone for treatment of pulmonary embolism. Studies have shown that systemic thrombolytic therapy decreases total mortality, recurrent thromboembolism, and pulmonary embolism-related mortality in the setting of a massive pulmonary embolism. ${ }^{21-23} \mathrm{How}-$ ever, thrombolytic therapy is associated with an increase of major and fatal bleeding, with the most devastating complication being intracranial hemorrhage. ${ }^{22,24,25}$ The Pulmonary Embolism Thrombolysis (PEITHO) trial showed that systemic thrombolysis reversed hemodynamic decompensation but increased major hemorrhage (6.3\%) and stroke $(2.4 \%)$ at 7 days. ${ }^{26}$ The International Cooperative Pulmonary Embolism Registry study evaluated the outcomes of patients with acute pulmonary embolism at 52 hospitals in 7 countries and demonstrated that intracranial hemorrhage may occur in up to $3 \%$ of patients who receive thrombolytic therapy. ${ }^{27}$ Although systemic thrombolysis is an approved treatment strategy, it carries significant risk, and alternative treatment approaches such as catheter-based or open surgical embolectomy have been suggested for high-risk patients.

For submassive pulmonary embolism characterized by the presence of RV strain while the patient is hemodynamically stable, treatment options and best clinical practice are the subject of considerable debate because significant mortality benefits have not been demonstrated with systemic thrombolysis. ${ }^{28}$ In patients with submassive pulmonary embolism, EkoSonic Endovascular System catheter-directed thrombolysis is an approved treatment methodology for adult patients, but it is not performed widely in pediatric patients, so experience in this setting is limited. ${ }^{29}$

The 2 classic trials that specify a dosing protocol and duration of treatment for ultrasound-assisted thrombolysis are the ULTIMA and the SEATTLE II trials. ${ }^{16,17}$ These studies were based on adult populations and used the EkoSonic Endovascular System. The ULTIMA trial (2014) used an r-tPA dose of $1 \mathrm{mg} / \mathrm{hr} \times 5$ hours that was decreased to $0.5 \mathrm{mg} / \mathrm{hr} \times 10$ hours. This trial demonstrated improvement in RV parameters without an increased risk of major bleeding or death. ${ }^{16}$ The SEATTLE II protocol called for an r-tPA dose of $1 \mathrm{mg} / \mathrm{hr}$ per device for 24 hours. The typical r-tPA infusion varied between 0.5 and $1.0 \mathrm{mg} / \mathrm{hr}$ depending on the size of the pulmonary embolism and the medical state, size, and age of the patient. The SEATTLE II study (2015) also showed improvement in RV function; however, 10\% of patients had major bleeding events. No patient experienced intracranial hemorrhage. ${ }^{17}$ A more recent study (2018) is the Optimum Duration of Acoustic Pulse Thrombolysis Procedure in Acute Intermediate-Risk Pulmonary Embolism (OPTALYSE PE) study that examined the optimal duration and dose of r-tPA in combination with EkoSonic Endovascular System catheter-directed thrombolysis in the treatment of acute submassive pulmonary embolism. The cohort that received the smallest dose and shortest duration (the 2-hour cohort that received $4 \mathrm{mg}$ of r-tPA per catheter) exhibited no bleeding events and had a $24 \%(P<0.0001)$ reduction in $\mathrm{RV} / \mathrm{LV}$ ratio; these findings were comparable to longer duration and higher dose cohorts. ${ }^{30}$

Mishra et al looked at the outcomes of catheter-directed thrombolysis compared to systemic thrombolysis in the setting of massive pulmonary embolism. ${ }^{31}$ They performed a propensity score match analysis and found that the rates of in-hospital mortality, 30-day readmission, secondary outcomes, and major bleeding complications were lower in the catheter-directed thrombolysis cohort. The researchers concluded that catheter-directed thrombolysis was superior to systemic thrombolysis in patients with pulmonary embolism.

Arora and colleagues performed a similar analysis and found that in-hospital mortality, 30-day readmission, gastrointestinal bleeding, and intracranial bleeding were lower in the catheter-directed thrombolysis cohort than in the system thrombolysis cohort. ${ }^{32}$

Other studies have looked at local infusion of r-tPA without ultrasound assistance. Lin et al compared catheterdirected infusion with a simple catheter vs ultrasoundassisted catheter-directed thrombolysis using the EkoSonic Endovascular System. ${ }^{33}$ The EkoSonic treatment arm had higher thrombus resolution rates, faster mean time of thrombolysis, and lower hemorrhagic complications, but no difference in mortality was seen between the 2 treatment arms. A retrospective study by Graif et al examined the efficacy of the EkoSonic Endovascular System compared with pigtail catheter-directed thrombolysis in the treatment of pulmonary embolism. ${ }^{34}$ They found no significant difference in the change in pulmonary artery pressure and the Miller pulmonary embolism severity index between the 2 treatment groups. Graif et al showed that procedure and fluoroscopy time for EkoSonic catheter placement is longer than for the pigtail catheter, but infusion times are shorter and the r-tPA dose is lower.

Limited data compare the different catheter-based therapies in the treatment of acute pulmonary embolism. The Standard vs Ultrasound-Assisted Catheter Thrombolysis for Submassive Pulmonary Embolism (SUNSET sPE; NCT02758574) trial will compare standard catheter infusion of thrombolytic agent with ultrasound-assisted catheter infusion. ${ }^{35}$ The trial is currently ongoing.

Randomized controlled trials of catheter-directed thrombolysis in the pediatric population have not been performed. Experience has principally been based on 
retrospective studies and case reports. For example, Bavare et al conducted a retrospective study of 5 pediatric patients who received a thrombolytic infusion of $r$-tPA (0.75-2 $\mathrm{mg} / \mathrm{hr}$ per catheter port) for 24 hours, and the patients experienced complete or partial resolution of their pulmonary embolism. ${ }^{29}$ One patient with complete resolution underwent another successful EkoSonic Endovascular System catheter-directed thrombolysis after 4 months for recurrence.

The PESI and the sPESI have been shown to be useful stratification tools for patients with acute pulmonary embolism, but no stratification tools exist for children in the setting of an acute pulmonary embolism. Our patient had high PESI and SPESI scores that stratified him as high risk. Although these scoring systems are based on studies done in adult populations, we speculate that the same principles apply to children as well.

The failure of initial treatment course in our patient could be attributed to multiple factors. First, no uniform recommendations are available in terms of indications, drug of choice, route of administration, and dosing for pulmonary embolism in pediatric patients. As previously stated, protocols of thrombolytic therapy have been extrapolated from adult guidelines. Second, the fibrinolytic system in children is a dynamic, evolving system with unique features that markedly influence the response to thrombolytic agents. ${ }^{36}$ Tarango and Manco-Johnson suggest that fibrinolysis may be downregulated throughout childhood because endogenous IPA and plasminogen activator inhibitor-1 do not reach adult levels until late adolescence. ${ }^{37}$ Therefore, age-related changes in the fibrinolytic system may be clinically significant. Our initial treatment might have been suboptimal because of the low dose and/or low treatment duration. During the second course of treatment, we administered a higher amount of thrombolytic for an extended period that resulted in clinical and hemodynamic improvement.

Extended-duration catheter-directed thrombolysis would, however, increase the risk of bleeding complications. Although no data are available that quantify the risk, the decision for extended treatment should be made cautiously, assessing risk to benefit for the individual patient. Our case demonstrates that catheter-directed thrombolysis of submassive pulmonary embolism restored hemodynamic stability and thus may prove to be an effective alternative to treatment with systemic r-tPA or surgical therapy, even in the pediatric setting.

\section{CONCLUSION}

Acute treatment of pulmonary embolus is an evolving area in the field of cardiovascular disease. Our case illustrates that catheter-directed thrombolysis may be beneficial in pediatric patients who sustain a submassive pulmonary embolism. However, the fibrinolytic system in children is different than that in adults, and children may have differences in the rate of clot resorption. Randomized controlled clinical trials are needed to elucidate the optimal dose and duration of catheter-directed thrombolysis in children.

\section{ACKNOWLEDGMENTS}

The authors have no financial or proprietary interest in the subject matter of this article.

\section{REFERENCES}

1. Guzmán-García SA, Montalvo GA, García-Santos A, et al. Hereditary spherocytosis and venous thrombosis of atypical site: a triple hematologic disease? J Hematol Blood Disord. 2016 June;2(2):201.

2. Perrotta S, Gallagher PG, Mohandas N. Hereditary spherocytosis. Lancet. 2008 Oct 18;372(9647):1411-1426. doi: 10.1016/S0140-6736(08)61588-3.

3. Schilling RF. Risks and benefits of splenectomy versus no splenectomy for hereditary spherocytosis-a personal view. $\mathrm{Br}$ J Haematol. 2009 Jun;145(6):728-732. doi: 10.1111/j.1365-2141.2009.07694.x.

4. Waheed W, Varigonda AL, Holmes CE, Trevino C, Borden NM, Pendlebury W. Spinal cord infarction in a patient with hereditary spherocytosis: a case report and discussion. Case Rep Neurol Med. 2016;2016:7024120. doi: 10.1155/2016/7024120.

5. Lopes B, Almeida L, Vicente A, et al. Extensive portal and superior mesenteric vein thrombosis after splenectomy in a patient with hereditary spherocytosis. $J$ Hematol. 2013;2(2):79-84.

6. Aggarwal A, Sharma V, Dwivedi S. Pulmonary thromboembolism following splenectomy in hereditary spherocytosis. J Med Cases. 2010;1 (1):14-15. doi: 10.4021/jmc2010.06.102e.

7. Stein PD, Kayali F, Olson RE. Incidence of venous thromboembolism in infants and children: data from the National Hospital Discharge Survey. J Pediatr. 2004;145(4):563-565. doi: 10.1016/j.jpeds.2004.06.021.

8. Aujesky D, Obrosky DS, Stone RA, et al. Derivation and validation of a prognostic model for pulmonary embolism. Am J Respir Crit Care Med. 2005 Oct 15;172(8):1041-1046. doi: 10.1164/rccm.200506-8620C.

9. Jimenez D, Aujesky D, Moores L, et al. Simplification of the pulmonary embolism severity index for prognostication in patients with acute symptomatic pulmonary embolism. Arch Intern Med. 2010;170(15):1383-1389.

10. Babyn PS, Gahunia HK, Massicotte P. Pulmonary thromboembolism in children. Pediatr Radiol. 2005;35(3):258-274.

11. Activase [package insert]. San Francisco, CA: Genentech, Inc.; 2015. www.accessdata.fda.gov/drugsatfda_docs/label/ 2015/103172s5203lbl.pdf. Accessed July 9, 2019.

12. Avgerinos ED, Chaer RA. Catheter-directed interventions for acute pulmonary embolism. J Vasc Surg. 2015 Feb;61(2):559-565. doi: 10.1016/j.jvs.2014.10.036.

13. Jaff MR, McMurtry MS, Archer SL, et al. Management of massive and submassive pulmonary embolism, iliofemoral deep vein thrombosis, and chronic thromboembolic pulmonary hypertension: a scientific statement from the American Heart Association. Circulation. 2011 Apr 26;123(16):1788-1830. doi: 10.1161/CIR.0b013e318214914f.

14. Kuo WT, Banerjee A, Kim PS, et al. Pulmonary embolism response to fragmentation, embolectomy, and catheter thrombolysis (PERFECT): initial results from a prospective multicenter registry. Chest. 2015 Sep;148(3):667-673. doi: 10.1378/chest.15-0119.

15. Bagla S, Smirniotopoulos JB, van Breda A, Sheridan MJ, Sterling KM. Ultrasound-accelerated catheter-directed thrombolysis for acute submassive pulmonary embolism. J Vasc Interv Radiol. 2015;26(7):1001-1006.

16. Kucher N, Boekstegers $P$, Müller O, et al. Randomized controlled trial of ultrasound-assisted catheter-directed thrombolysis for acute intermediate-risk pulmonary embolism. 
Circulation. 2014 Jan 28;129(4):479-486. doi: 10.1161/CIRCULATIONAHA.113.005544.

17. Piazza G, Hohlfelder B, Jaff MR, et al.; SEATTLE II Investigators. A prospective, single-arm, multicenter trial of ultrasound-facilitated, catheter-directed, low-dose fibrinolysis for acute massive and submassive pulmonary embolism: the SEATTLE II study. JACC Cardiovasc Interv. 2015 Aug 24;8(10):1382-1392. doi: 10.1016/j.jcin.2015.04.020.

18. Perkins LA, Jones SF, Bhargava RS. Dural venous thrombosis following splenectomy in a patient with hereditary spherocytosis. South Med J. 2009 May;102(5):542-545. doi: 10.1097/SMJ.0b013e31819e90b5.

19. Troendle SB, Adix L, Crary SE, Buchanan GR. Laboratory markers of thrombosis risk in children with hereditary spherocytosis. Pediatr Blood Cancer. 2007;49(6): 781-785.

20. Byrnes JR, Wolberg AS. Red blood cells in thrombosis. Blood. 2017 Oct 19;130(16):1795-1799. doi: 10.1182/blood-2017-03-745349.

21. Wan S, Quinlan DJ, Agnelli G, Eikelboom JW. Thrombolysis compared with heparin for the initial treatment of pulmonary embolism: a meta-analysis of the randomized controlled trials. Circulation. 2004 Aug 10;110(6):744-749. doi: 10.1161/01.CIR.0000137826.09715.9C.

22. Jerjes-Sanchez C, Ramírez-Rivera A, de Lourdes García M, et al. Streptokinase and heparin versus heparin alone in massive pulmonary embolism: a randomized controlled trial. J Thromb Thrombolysis. 1995;2(3):227-229.

23. Dotter CT, Seaman AJ, Rösch J, Porter JM. Streptokinase and heparin in the treatment of pulmonary embolism: a randomized comparison. Vasc Surg. 1979;13(1):42-52.

24. Ly B, Arnesen $\mathrm{H}$, Eie H, Hol R. A controlled clinical trial of streptokinase and heparin in the treatment of major pulmonary embolism. Acta Med Scand. 1978;203(6):465-470.

25. Marti C, John G, Konstantinides S, et al. Systemic thrombolytic therapy for acute pulmonary embolism: a systematic review and meta-analysis. Eur Heart J. 2015;36(10):605-614. doi: 10.1093/eurheartj/ehu218.

26. Meyer G, Vicaut E, Danays T, et al.; PEITHO Investigators. Fibrinolysis for patients with intermediate-risk pulmonary embolism. N Engl J Med. 2014 Apr 10;370(15):1402-1411. doi: 10.1056/NEJMoa1302097.

27. Goldhaber SZ, Visani L, De Rosa M. Acute pulmonary embolism: clinical outcomes in the International Cooperative
Pulmonary Embolism Registry (ICOPER). Lancet. 1999 Apr 24;353:1386-1389.

28. Konstantinides S, Goldhaber SZ. Pulmonary embolism: risk assessment and management. Euro Heart J. 2012 Dec;33(24):3014-3022. doi: 10.1093/eurheartj/ehs258.

29. Bavare AC, Naik SX, Lin PH, et al. Catheter-directed thrombolysis for severe pulmonary embolism in pediatric patients. Ann Vasc Surg. 2014 Oct;28(7):1794. e1-e7. doi: 10.1016/j.avsg.2014.03.016.

30. Tapson VF, Sterling K, Jones N, et al. A randomized trial of the optimum duration of acoustic pulse thrombolysis procedure in acute intermediate-risk pulmonary embolism: the OPTALYSE PE trial. JACC Cardiovasc Interv. 2018 Jul 23;11(14):1401-1410. doi: 10.1016/j.jcin.2018.04.008.

31. Mishra A, Kamboj S, Shah P, et al. Comparison of catheter-directed thrombolysis vs systemic thrombolysis in pulmonary embolism: a propensity score match analysis. Chest. 2017 Oct;152(4):A1047. doi: 10.1016/j.chest.2017.08.1080.

32. Arora S, Panaich SS, Ainani N, et al. Comparison of in-hospital outcomes and readmission rates in acute pulmonary embolism between systemic and catheter-directed thrombolysis (from the National Readmission Database). Am J Cardiol. 2017 Nov 1;120(9):1653-1661. doi: 10.1016/j.amjcard.2017.07.066.

33. Lin PH, Annambhotla S, Bechara CF, et al. Comparison of percutaneous ultrasound-accelerated thrombolysis versus catheter-directed thrombolysis in patients with acute massive pulmonary embolism. Vascular. 2009 Nov-Dec;17 Suppl 3:S137-S147.

34. Graif A, Niesen TJ, Gakhal M, et al. Comparison of percutaneous ultrasound-accelerated endovascular thrombolysis (EKOS) with pigtail catheter directed thrombolysis for the treatment of acute pulmonary embolism. J Vasc Interv Radiol. 2015;2(26):S75. doi: 10.1016/j.jvir.2014.12.209.

35. Avgerinos ED, Mohapatra A, Rivera-Lebron B, et al.; PERT Consortium. Design and rationale of a randomized trial comparing standard versus ultrasound-assisted thrombolysis for submassive pulmonary embolism. J Vasc Surg Venous Lymphat Disord. 2018 Jan;6(1):126-132. doi: 10.1016/j.jvsv.2017.09.004.

36. Albisetti M. The fibrinolytic system in children. Semin Thromb Hemost. 2003 Aug;29(4):339-348. doi: 10.1055/s-2003-42585.

37. Tarango C, Manco-Johnson MJ. Pediatric thrombolysis: a practical approach. Front Pediatr. 2017 Dec 6;5:260. doi: 10.3389/fped.2017.00260.

This article meets the Accreditation Council for Graduate Medical Education and the American Board of Medical Specialties Maintenance of Certification competencies for Patient Care and Medical Knowledge. 